\title{
Post-mortem whole-exome sequencing (WES) with a focus on cardiac disease-associated genes in five young sudden unexplained death (SUD) cases
}

\author{
Jacqueline Neubauer $^{1}$ - Cordula Haas $^{1}$ - Christine Bartsch ${ }^{1}$. \\ Argelia Medeiros-Domingo ${ }^{2} \cdot$ Wolfgang Berger ${ }^{3,4,5}$
}

Received: 21 September 2015 / Accepted: 13 January 2016/Published online: 4 February 2016

(C) Springer-Verlag Berlin Heidelberg 2016

\begin{abstract}
Sudden death of healthy young adults in the absence of any medical reason is generally categorised as autopsy-negative sudden unexplained death (SUD). Approximately $30 \%$ of all SUD cases can be explained by lethal sequence variants in cardiac genes causing disturbed ion channel functions (channelopathies) or minimal structural heart abnormalities (cardiomyopathies). The aim of this study was to perform whole-exome sequencing (WES) in five young SUD cases in order to identify potentially disease-causing mutations with a focus on 184 genes associated with cardiac diseases or sudden death. WES analysis enabled the identification of damaging-predicted cardiac sequence alterations in three out of five SUD cases. Two SUD victims carried disease-causing variants in long QT syndrome (LQTS)-associated genes $(\mathrm{KCNH} 2, \mathrm{SCN} 5 \mathrm{~A})$. In a third case, WES identified variants in two genes involved in mitral valve prolapse
\end{abstract}

Electronic supplementary material The online version of this article (doi:10.1007/s00414-016-1317-4) contains supplementary material, which is available to authorized users.

Jacqueline Neubauer

jacqueline.neubauer@irm.uzh.ch

1 Zurich Institute of Forensic Medicine, University of Zurich, Winterthurerstrasse 190/52, 8057 Zurich, Switzerland

2 Department of Cardiology, Inselspital, University Hospital, Bern, Switzerland

3 Institute of Medical Molecular Genetics, University of Zurich, Schlieren, Switzerland

4 Center for Integrative Human Physiology (ZIHP), University of Zurich, Zurich, Switzerland

5 Neuroscience Center (ZNZ), University and ETH Zurich, Zurich, Switzerland and thoracic aortic aneurism (DCHS1, TGF 32$)$. The genome of a fourth case carried several minor variants involved in arrhythmia pointing to a multigene influence that might have contributed to sudden death. Our results confirm that postmortem genetic testing in SUD cases in addition to the conventional autopsy can help to identify familial cardiac diseases and can contribute to the identification of genetic risk factors for sudden death.

Keywords Sudden unexplained death · Cardiac diseases . Molecular autopsy $\cdot$ Whole-exome sequencing

\section{Introduction}

Sudden death (SD) of a previously healthy adolescent or young adult is a tragic and distressing event for those left behind. Depending on the underlying cause of death, SD can be divided into sudden cardiac death (SCD) or sudden death due to non-cardiac causes such as pulmonary embolism, metabolic, neurological, or infectious conditions [1, 2]. In elderly persons, SCD is mainly caused by coronary artery disease (CAD), whereas in young people (between 1 and 39 years of age), a complete post-mortem examination fails to reveal a cause of death in up to $30 \%$ of all cases $[3,4]$. These are generally designated as autopsy-negative sudden unexplained death (SUD) cases. Although SCD is one of the major causes of mortality worldwide, the exact incidence of SUD in young individuals remains unknown in most countries [5, 6].

It is estimated that up to $30 \%$ of all SUD cases carry sequence variants in known cardiac genes causing lethal cardiac arrhythmia in a morphological normal heart $[3,7,8]$. Channelopathies primarily affect the heart rhythm and cardiac electrical conduction including cardiac diseases such as long QT syndrome (LQTS), short QT syndrome (SQTS), 
catecholaminergic polymorphic ventricular tachycardia (CPVT), Brugada syndrome (BrS), and idiopathic ventricular fibrillation (IVF) [9]. Cardiomyopathies on the other hand refer to myocardial disorders characterised by a structural and functional abnormal heart muscle [10]. The most common form is hypertrophic cardiomyopathy (HCM) followed by other cardiomyopathies such as arrhythmogenic right ventricular cardiomyopathy (ARVC), idiopathic dilated cardiomyopathy (DCM), and left ventricular non-compaction cardiomyopathy (LVNC) [11].

Post-mortem genetic testing by using specific gene panels or whole-exome sequencing (WES) represents an efficient tool to elucidate potential disease-causing mechanisms, which remained undetected during autopsy $[8,12,13]$. The exome represents approximately $1-2 \%$ of the human genome, but it accounts for over $85 \%$ of all mutations that have been identified in Mendelian disorders [14]. Loporcaro et al. recently published a first proof-of-principle case report of a WESbased comprehensive molecular autopsy of an otherwise healthy 16-year-old SUDY (SUD in the young) victim, where they identified a pathogenic $M Y H 7$ mutation, previously described in familial HCM, sudden death, and impaired MHC- $\beta$ actin-translocating and actin-activated ATPase activity [12]. Further studies described the detection of eight ultra-rare variants in seven of 14 Caucasian SUDY victims by focusing on 117 sudden death-susceptibility genes and the identification of likely pathogenic variants in 34 genes in $20 \%$ of forensic SUD cases $[8,15]$. Post-mortem genetic analysis of SUD victims may not only enable an accurate determination of cause and manner of death, but may also have significant impact for affected living first-degree family members in whom deathpredisposing disorders had remained unrecognised so far $[16$, 17].

At the Zurich Institute of Forensic Medicine (ZIFM), University of Zurich, Switzerland, approximately 10 sudden death cases in young individuals are reported annually to show no or only minor morphological abnormalities during autopsy, leaving those left behind without any explanation about the exact cause of death. WES is not substantially more expensive than a targeted approach using a gene panel, but allows additional examination of new candidate genes in case of a negative result within the predefined gene list. In this study, we performed WES analyses in five unrelated SUD cases by focusing on 184 cardiac disease-associated genes. We wanted to test whether such a procedure could be implemented as a standard operating procedure to investigate or uncover lethal cardiac diseases.

\section{Material and methods}

Five unrelated sudden unexplained death cases (four females and one male) were collected between March and
May 2012 at the ZIFM, Switzerland. All SUD cases were examined according to standard forensic procedures, including death scene investigation, a complete autopsy examination with pharmacological-toxicological and histopathological screening, and review of the clinical history. Inclusion criteria were (1) younger than 40 years of age, (2) only minor abnormalities in the heart, and (3) suspicion of an inherited cardiac disease or underlying cardiac arrhythmia contributing to sudden death. DNA of firstdegree relatives of the five SUD cases was not available for co-segregation analysis.

\section{Ethical approval}

Ethical approval for this study was provided by the local committee in Zurich (KEK-ZH-Nr. 2013-0086), and the study was conducted in full conformance with Swiss laws and regulations.

\section{DNA sample collection and extraction}

Five-milliliter EDTA blood samples from the five SUD cases were collected during autopsy and were stored at $-20{ }^{\circ} \mathrm{C}$. Genomic DNA was extracted using the Gentra Puregene Blood Kit (Qiagen, Hombrechtikon, Switzerland) according to the manufacturer's protocol and quantified using the NanoDrop2000 (Fischer Scientific, Wohlen, Switzerland).

\section{Exome sequencing}

Exome capture, sequencing as well as sequence alignment and variant calling were performed at Atlas Biolabs (Berlin, Germany). Nimblegen SeqCap EZ Human Exome Library v.2.0 (Roche NimbleGen Inc., Madison, USA) was used for library preparation, and sequencing was done on the Illumina HiSeq2000 platform (Illumina Inc., San Diego, USA) generating $2 \times 100 \mathrm{bp}$ paired-end reads [18]. Alignment of sequence reads, indexing of the reference genome (hg19), and variant calling and annotation was done with a pipeline based on BWA [19], Samtools [20], and Picard and Annovar [21].

\section{Filtering steps and data analysis}

Variant filtering included a gene panel of 184 genes associated with cardiac diseases or cardiac death (Supp. Table S1). Annotation of these genes was conducted with the software Alamut HT version 1.1.7 (Interactive Biosoftware, Rouen, France). Output results were imported into an excel-sheet for further analysis. At first, variants were checked manually if they are disease-associated according to entries from the Human Gene Mutation 
Fig. 1 Filtering flow chart. Whole-exome sequencing data were filtered for 184 genes associated with cardiac diseases and/or sudden death. At first, variants were checked in HGMD $^{\circledR}$ (Human Genome Mutation Database Professional) for reported variant classes (filter $2 A$ ). The filtering steps for all other variants focused on a global MAF less than 0.02 (filter 2B); exonic and splice site variants (filter 3); InDels, missense, and nonsense variants (filter 4); and deleterious or disease-causing protein prediction (filter 5)

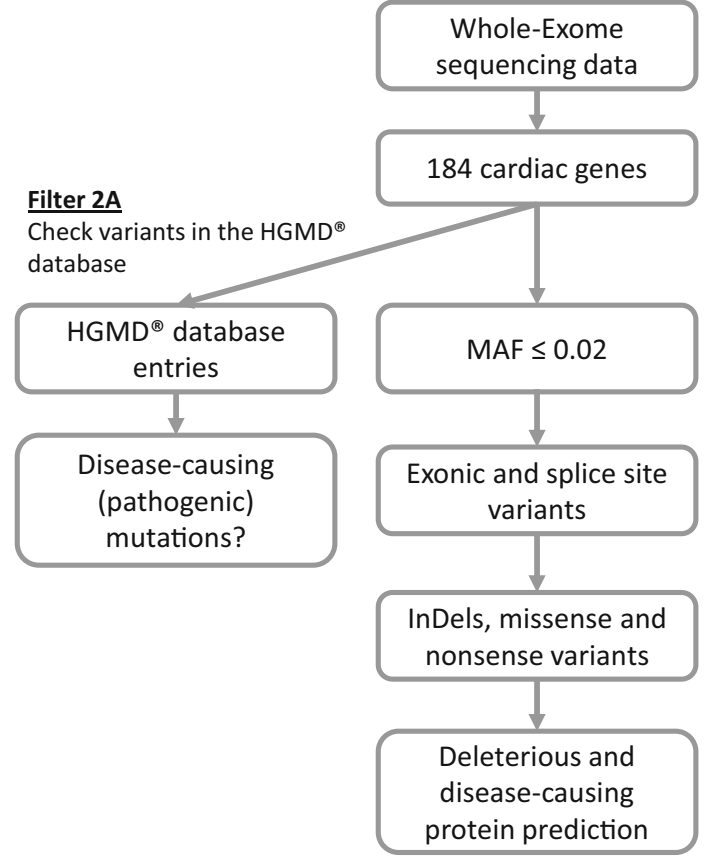

Filter 1

Focus on 184 cardiac genes associated with cardiac diseases and/or sudden death

\section{Filter 2B}

Focus on variants with a global minor allele frequency $\leq 0.02$ (dbSNP)

\section{Filter 3}

Focus on exonic and splice site $(+1,+5)$ variants

\section{Filter 4}

Focus on InDels, missense and nonsense variants

\section{Filter $\mathbf{5}$}

Check if variants are bad (MAPP) deleterious (SIFT), damaging (PolyPhen2) or disease-causing (MutationTaster)
Database Professional (HGMD $\left.{ }^{\circledR}\right)$ (BIOBASE GmbH, Wolfenbüttel, Germany) (Fig. 1). To include known modulator and risk factor variants into data analysis, $\mathrm{HGMD}^{\circledR}$-reported variants were not filtered for a threshold minor allele frequency (MAF). The filtering steps for all other variants focused on (1) a global MAF value less than or equal to 0.02 (based on dbSNP); (2) exonic and splice site $(+1,+5)$ variants; (3) exonic InDels, missense, and nonsense variants; and (4) deleterious or damaging protein predicted functions according to the following in silico predictive algorithms: MAPP (multivariate analysis of protein polymorphism prediction), SIFT (sorting intolerant from tolerant prediction), PolyPhen2, AGVGD (align Grantham-variation Grantham-deviation), and MutationTaster. Sequence alterations at the 5' donor splice sites +1 and +5 were included into data analysis, because variants at these positions are thought to significantly reduce the pairing of the donor splice site with the complementary site in the small nuclear ribonucleoprotein particle U1snRNP, which is one of the first steps in the complex process of messenger RNA (mRNA) splicing [22]. Alamut Visual version 2.4.7 (Interactive Biosoftware, Rouen, France) was used to visualise the coverage of variants and to review conservation of the variants across a variety of species (based on Ensembl Genome Browser, http://ensembl.org). European allelic frequencies were examined in dbSNP at NCBI (www.ncbi. nlm.nih.gov/projects/SNP/), Ensembl, and in the exome aggregation consortium database (ExAC) (http://exac. broadinstitute.org/).

Possible disease-causing variants must be absent or rare in public databases (dbSNP, ExAC) or in 288 analysed controls (177 males, 111 females) from the general Swiss population.
As a final step, genes identified with disease-causing variants were analysed for splice site and/or synonymous variants.

\section{Confirmation and validation of variants}

Potential disease-causing variants found in whole-exome sequencing data of the five SUD cases were confirmed and screened in 288 controls by using standard polymerase chain reaction (PCR) and Sanger sequencing methods (primer sequences available on request). Sequence data were aligned to the reference sequence with the SeqScape ${ }^{\circledR}$ software version v2.6 (Life Technologies, Rotkreuz, Switzerland) (Supplementary data).

\section{Results}

Whole-exome sequencing was performed in five unrelated SUD cases. Data were analysed with respect to sequence variants with a focus on 184 genes previously associated with cardiac diseases or cardiac death. WES yielded an average of 87433402.8 total reads, and $87.44 \%$ of the target positions were covered at least 30 times (Table 1). Within the exome sequencing data, the five cases had on average $40658 \pm 613.2$ single nucleotide variants (SNV) and insertion/deletions (InDels) causing amino acid changes (missense variants) on average in $10788.4 \pm 162.2$ variants. By focusing only on the cardiac gene panel, on average $633.2 \pm 10.6$ variants were obtained per case. After all filtering steps, $23.4 \pm 6.3$ variants were used for further manual evaluation. 
Table 1 Summary of WES data

\begin{tabular}{llllll}
\hline & Case I & Case II & Case III & Case IV & Case V \\
\hline Raw data & & & & & \\
Total reads & $81^{\prime} 368^{\prime} 374$ & $99^{\prime} 487^{\prime} 232$ & $97^{\prime} 357^{\prime} 972$ & $84^{\prime} 440^{\prime} 830$ & $74^{\prime} 512^{\prime} 606$ \\
Variants covered $>10 \times(\%)$ & 97.3 & 97.6 & 97.6 & 97.4 & 97 \\
Variants covered $>30 \times(\%)$ & 86.6 & 89.6 & 89.4 & 87.5 & 84.1 \\
Total number of SNV/InDels & $40^{\prime} 673$ & $40^{\prime} 647$ & $40^{\prime} 039$ & $41^{\prime} 648$ & $40^{\prime} 283$ \\
Exonic variants & $23^{\prime} 041$ & $22^{\prime} 908$ & $22^{\prime} 554$ & $23^{\prime} 236$ & $22^{\prime} 621$ \\
Missense variants & $10^{\prime} 431$ & $10^{\prime} 254$ & $10^{\prime} 155$ & $10^{\prime} 975$ & $10^{\prime} 239$ \\
Not reported in dbSNP & 1743 & 958 & 859 & 1145 & 906 \\
Frameshift InDels & 170 & 160 & 154 & 155 & 140 \\
Stopgain & 112 & 105 & 92 & 93 & 83 \\
Stoploss & 22 & 22 & 19 & 17 & 15 \\
Splicing & 119 & 117 & 119 & 120 & 112 \\
Variants in 184 cardiac genes & & & & & 631 \\
Total variants & 627 & 645 & 620 & 643 & 19 \\
HGMD ${ }^{\circledR}$ variants & 16 & 16 & 21 & 24 & 133 \\
MAF $\leq 0.02$ & 130 & 145 & 150 & 137 & 68 \\
Exonic/splice site variants & 75 & 77 & 84 & 87 & 17 \\
Missense variants & 22 & 23 & 23 & 21 & 0 \\
Not reported in dbSNP & 6 & 4 & 2 & 3 & 1 \\
Deletions & 2 & 0 & 0 & 1 & 0 \\
Insertion & 0 & 0 & 0 & 2 & 0 \\
Nonsense & 0 & 0 & & & \\
\hline
\end{tabular}

$M A F$ minor allele frequency, $S N V$ single nucleotide variants, InDels insertion/deletions

\section{Case I}

Case I was a 27-year-old woman (weight, $54.1 \mathrm{~kg}$; height, $172 \mathrm{~cm}$; European origin) found dead on a sofa by her roommate late in the evening. Autopsy investigations showed a normal heart size (330 g) according to Zeek [23], but a swollen and oedematous mitral valve with connective tissue changes in papillary muscles and thickening of tendinous chords (Supp. Fig. S1a). Histological examination of the mitral valve revealed thickening and mucoid swelling of the tissue with signs of collagenous destruction (Supp. Fig. S1b). Microbiological, virological, and pharmacological-toxicological screening results were all negative and showed no signs of an acute inflammation prior to death.

At least two potential disease-causing heterozygous variants were detected in the 27-year-old woman (Table 2). A missense variant was located in the dachsous cadherinrelated 1 gene (DCHS1; NM_003737.2: c.3415C > T: p.Arg1139Cys) with disease-causing protein function predictions and an allele frequency of $3.7 \mathrm{e}-05$ in the general population (ExAC). The second variant was a one nucleotide, frame-shifting deletion in the transforming growth factor beta 2 gene (TGFß2; NM_001135599.2: c.440delC: p.Pro.147 Argfs*27) leading to a truncated protein, not previously described in respective databases or in the scientific literature.
The ancestral alleles of both variants were highly conserved during evolution.

\section{Case II}

Case II was a 30-year-old woman (weight, $51.6 \mathrm{~kg}$; height, $166 \mathrm{~cm}$; European origin). She collapsed in the bathroom and was brought into her bed, where she died shortly afterwards. She had severe depression and was alcohol-dependent. Chemical screening tests detected a massively increased amount of butyric acid (almost a lethal concentration of $2500 \mu \mathrm{mol} / \mathrm{L}$ ) and further pathological metabolic products such as acetone. Pharmacologicaltoxicological screening tests detected therapeutic concentrations of benzodiazepines in the peripheral blood. Autopsy investigations showed a heart weight of $390 \mathrm{~g}$ (24\% enlarged according to Zeek) with an adipose right ventricle and focally enlarged myocytes.

Within the 184 cardiac genes, we found at least two important possibly phenotype-relevant heterozygous missense variants (Table 2). One variant (rs76906304) was located in the gap junction protein delta 4 gene (GJD4; NM_153368.2: c. $259 \mathrm{C}>\mathrm{T}$ : p.Leu87Phe). The second variant was detected in the junctophilin type 2 gene (JPH2; NM_020433.4: c.1013A > G: p.Glu338Gly), not previously described in the 


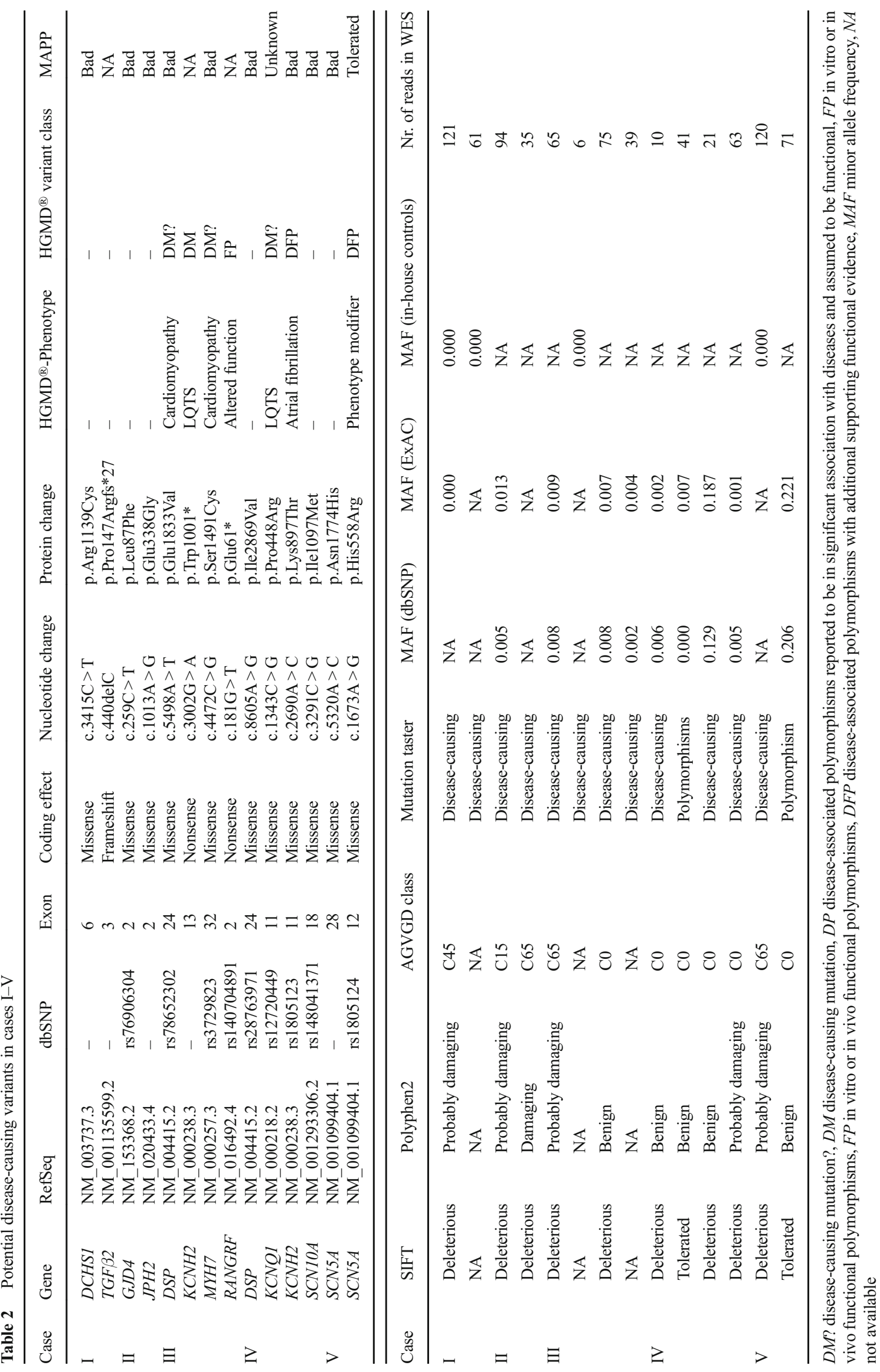


literature or public databases. Both variants were predicted to be disease-causing/damaging according to the in silico predictive algorithms.

\section{Case III}

Case III was a 31-year-old man (weight, $87.6 \mathrm{~kg}$; height, $186 \mathrm{~cm}$; European origin) who was found dead sitting on the toilet in his apartment. He worked in a restaurant kitchen, but did not appear to his working shift although he was seen shortly before and was considered to be reliable. On the evening before, he has attended a concert with some colleagues where they allegedly consumed different drugs. The autopsy showed a moderate enlarged heart (390 g) without further morphological abnormalities. Post-mortem pharmacological-toxicological screening results were positive for cannabis and amphetamine. Further investigations of the medical history revealed a long QT syndrome (LQTS) diagnosis which was already detected in his adolescence based on electrocardiography (ECG) results (Supp. Fig. S2). His physician recommended an annual medical check including an ECG stress test, but he did not prescribe any medications. Two of the deceased's brothers and mother had the same diagnosis of LQTS, but without any implications so far (Supp. Fig. S3).

Within the cardiac gene panel, we found two nonsense alterations and two missense variants (Table 2). One stop variant (rs121912509) was detected in the gene encoding the potassium voltage-gated channel, subfamily $\mathrm{H}$, member 2 (KCHN2; NM_000238.3: c.3002G > A: p.Trp1001*), reported as a LQT2 disease-causing mutation according to HGMD $^{\circledR}$. Due to the low number of reads (coverage $=6$ ) at this position, Sanger sequencing was performed to confirm the variant (supplementary data). A second stop variant (rs140704891) was identified in the RAN guanine nucleotide release factor gene (RANGRF; NM_016492.4: c.181G > T: p.glu61*) and is classified as an in vitro or in vivo functional polymorphism in $\mathrm{HGMD}^{\circledR}$. Two potentially disease-relevant heterozygous missense variants were located in the desmoplakin gene (DSP; NM_004415.2: rs78652302: c.5498A > T: p.Glu1833Val) and in the myosin heavy chain 7 gene (MYH7; NM_000257.2: rs3729823: c. $4472 \mathrm{C}>\mathrm{G}$ : p.Ser1491Cys). Both variants were described as possible disease-causing mutations according to $\mathrm{HGMD}^{\circledR}$ (rs78652302: dilated cardiomyopathy and rs3729823: hypertrophic cardiomyopathy), and protein effects were predicted to be deleterious (SIFT), probably damaging (polyphen2), and disease-causing (MutationTaster). MAF for both variants is $\sim 1 \%$ in the general population, but shows a variable frequency within the European population (Ensembl: rs78652302: 13\%T and rs3729823: 0-3\%G).

\section{Case IV}

Case IV was a 38 -year-old woman (weight, $58.8 \mathrm{~kg}$; height, $162 \mathrm{~cm}$; Tibetan origin). She was found dead in the morning by her 10-year-old daughter who was sleeping in the same bed. Since several weeks, the woman complained about the air conditioner at her new job as she had a cold, but was otherwise healthy. Autopsy findings showed a normal heart size $(250 \mathrm{~g})$ and a slightly fatty infiltrated right ventricle without any further morphological abnormalities. Family history was negative for cardiac diseases or sudden death.

Genetic testing identified no rare disease-causing sequence variants within the cardiac gene panel, but the woman had several minor variants which probably affect function (Table 2). One heterozygous missense variant was located in $\mathrm{KCNH} 2$ (rs1805123: c.2690 T>G: p.Lys897Thr) and is associated with atrial fibrillation and drug-induced arrhythmia according to $\mathrm{HGMD}^{\circledR}$. Although the minor allele is common in the European population $(20 \% \mathrm{G})$, the variant is significantly less common in the Asian population (4\%G). Further heterozygous missense variants were located in DSP (rs28763971: c. $8605 \mathrm{~A}>\mathrm{G}:$ p.Ille2869Val), in the potassium voltage-gated channel, KQT-type gene (KCNQ1; NM_000218.2: rs12720449: c.1343C > G: p.Pro448Arg), and in the sodium channel type 10 gene (SCN10A; NM_006514.2: rs148041371: c.3291C > G: p.Ille1097Met). Although all three sequence variants are very rare in the European population, they are much more common in the Asian population (Ensembl: rs28763971: 2\%G; rs12720449: 11\%G; rs148041371: $2 \% \mathrm{G})$.

\section{Case V}

Case V was a 19-year-old woman (weight, $68.4 \mathrm{~kg}$; height, $170 \mathrm{~cm}$; European origin) who gave birth to her first child 2 months before she died. She had a minor cough, but was otherwise healthy when she suddenly collapsed at her home. The ambulance transferred her to the hospital where lifesustaining measures were stopped after the diagnosis of myocardial infarction and irreversible brain damage. Autopsy investigation showed a broadening and fatty infiltrated right ventricle possibly indicating ARVC (Supp. Fig. S4 a, b), and the heart was slightly enlarged (300 g). Pharmacologicaltoxicological screening tests were negative for drugs and alcohol. Further investigations of the family history showed that a niece of the deceased died suddenly at the age of 11 during sports, and the grandfather died suddenly while sweeping snow at the of age of 77 (Supp. Fig.S5). Furthermore, her mother had minimal prolongation of the heart rate corrected QT intervals (QTc) of 460-480 ms [24]; however, she did not follow further medical examinations. The toddler of this young woman has been under medical observation, a QTc 
prolongation ( $495 \mathrm{~ms}$ ) has been detected during sleep, but the echocardiogram was normal.

Analysis of sequence variants within the 184 cardiac genes revealed two heterozygous variants in the voltage-gated sodium channel type V, alpha subunit (SCN5A) (Table 2). One missense variant was located in exon 28 (NM 001099404.1: c. $5320 \mathrm{~A}>$ C, p.Asn1774His) and was not previously reported in the literature and in public databases. A second missense variant was located in exon 12 (NM_001099404.1: rs 1805124 , c. $1673 \mathrm{~A}>\mathrm{G}$, p.His558Arg) and is described as disease-associated polymorphism with additional supporting functional evidence according to $\mathrm{HGMD}^{\circledR}$. However, prediction algorithms described the variant as tolerated, and MAF is $21 \%$ in the general population.

\section{Discussion}

Molecular testing in autopsy-negative death cases can identify the underlying mechanisms for sudden death and may represent an efficient tool in combination with standard forensic investigations to identify or confirm the suspicion of an inherited sudden death-predisposing disorder [8].

In this study, genetic testing by using WES enabled the identification of potential lethal cardiac disease-associated alterations in three out of five SUD victims (Table 3). A fourth case carried several minor variants pointing to a multigene influence instead of a strong autosomal-dominant sequence variant which could explain the phenotype.

Case I had one missense variant in DCHSI (p.Arg1139Cys) and a one nucleotide frame-shift deletion in $T G F \beta 2$ (p.Pro.147 fs*27). Sequence variants in both genes were already reported in mitral valve prolapse (MVP), which is a valvular heart disease characterised by the displacement of an abnormally thickened mitral valve leaflet into the left atrium during systole leading to serious complications, including arrhythmia, heart failure, and sudden death [25]. Reanalysis of the case report showed that the young woman had scoliosis and was very tall with long, slender limbs and fingers. Point mutations and whole-gene deletions/duplications in $T G F \beta 2$ are reported in patients with Loeys-Dietz syndrome (LDS), familial thoracic aortic aneurysms, and acute aortic dissection (TAAD) associated with mild systemic features of Marfan syndrome (MFS) [26]. Phenotypical features and genetic testing strongly indicated that the young woman had an underlying connective tissue disease, obviously not diagnosed during her lifetime, which might have contributed to mitral valve prolapse leading to the sudden death of this young woman.

WES analysis in case II identified two variants in GJD4 (p.Leu87Phe) and JPH2 (p.Glu338Gly). Connexins, such as GJD4, are transmembrane proteins involved in the formation of gap junction channels in the heart. Alterations in the connexin family are associated with cardiac arrhythmias and sudden infant death syndrome (SIDS) [27, 28]. Junctophilin type 2 is a cardiac member of the junctional membrane complex proteins, and JPH2 knockout mice demonstrated embryonic lethality and irregular calcium handling suggesting a crucial role for junctophilin-2 in facilitating intracellular calcium release and cardiac contractility [29]. Sequence variants in $\mathrm{JPH} 2$ were reported in atrial fibrillation due to impaired ryanodine receptor $\mathrm{Ca}^{2+}$ channels (RyR2) stabilisation and in familial HCM [30]. Although the primary cause of death in this young woman was already diagnosed due to metabolic imbalance, the two GJD4 and JPH2 variants could have contributed to the morphological abnormalities in her heart, triggering the sudden death event during a critical time period.

Case III carried two nonsense sequence variants in RANGRF (p.glu61*) and KCNH2 (p.Trp1001*). RANGRF protein regulates the expression and function of SCN5A (Nav1.5) cardiac sodium channel. Electrophysiological studies in CHO-K1 cells demonstrated that the p.Glu61* stop completely failed to increase the sodium channel current compared to wild-type and may increase the risk of arrhythmias [31]. The Nav1.5 interacting part of RANGRF is positioned in the amino acid range 65-186 and hence a truncated protein produced from the affected allele would presumably not bind to the Nav1.5 leading to a loss of function [32]. $\mathrm{KCNH} 2$ encodes the pore forming subunit of the rapidly activating delayed rectifier $\mathrm{K}^{+}$channel $\left(\mathrm{I}_{\mathrm{Kr}}\right)$ in the heart. Sequence variants are associated with delayed cardiac repolarization and prolonged QTc intervals leading to ventricular arrhythmias and sudden death [33]. The stop variant (c.3002C $>$ T; p.Trp1001*) affects the C-terminus region of the $\mathrm{KCNH} 2$ protein leading to assembly abnormality caused by a reduction of mutant mRNA transcripts by nonsense-mediated mRNA decay [34]. The p.Trp1001* could be the major underlying pathogenic sequence alteration causing QTc interval prolongation in this young man, confirming the ECG-based clinical diagnosis of LQTS. In addition, QTc interval prolongation is described in patients taking amphetamine-type stimulants [35], also detected in therapeutic concentration in this 31year-old man.

Genetic testing in case IV identified one variant in $\mathrm{KCNH} 2$ (p.Lys897Thr) and several borderline variants in arrhythmiaassociated genes (DSP: p.Ille2869Val; KCNQ1: p.Pro448Arg; SCN10A: p.Ille1097Met). Although sequence variants in $\mathrm{KCNH} 2$ are described in drug-induced QTc prolongation [36], we have no evidence if the woman had taken any medications against her cold prior to death. The common p.Lys897Thr is associated with an increased risk of developing torsade de pointes in the subacute phase of myocardial infarction and is described as a genetic modifier of latent congenital LQTS $[37,38]$. Furthermore, cases with multiple borderline variants are more susceptible to develop diseases, even in the absence of strong dominant mutations that explain the phenotype. One major problem during data analysis was that 


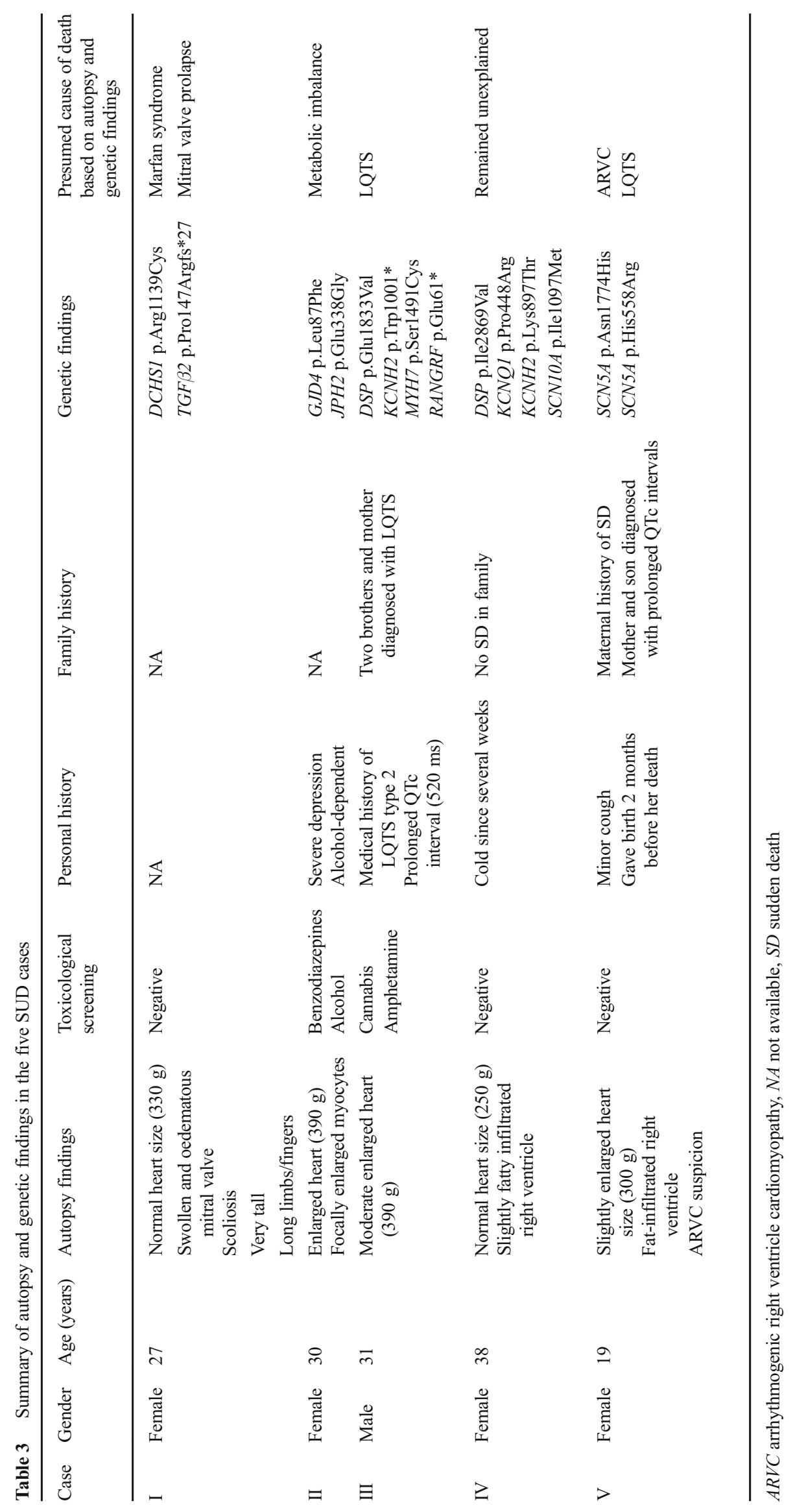


the allelic frequencies of Tibetan origin were not available in public databases, complicating accurate interpretation of the found sequence variants.

Case V carried a missense variant in SCN5A (p. Asn1774His) not previously reported in public databases. Kato et al. described a 1-day-old boy with prolonged QTc intervals $(680 \mathrm{~ms})$ having asparagine to aspartic acid replacement at the same position (rs199473633, c.5320 T>C, p.Asn1774Asp), and his brother died suddenly in infancy [39]. A functional assay demonstrated that p.Asn1774Asp channels carried significantly larger late components of sodium current than wild-type cells. The same amino acid replacement (p.Asn1774Asp) was also reported in a male foetus, which showed typical clinical signs of LQT3 (convulsion, ventricular tachycardia, torsade de pointes, atrioventricular block, and QTc intervals of $670 \mathrm{~ms}$ ) [40]. To investigate the consequences of the asparagine to histidine amino acid change found in this young woman, functional studies are required in order to study the biological relevance of this substitution. The second missense variant in SCN5A (p.His558Arg) is described as phenotype modifier in LQTS and might amplify or modulate the functional electrophysiological impairment of genetic variants located in the same gene [41, 42]. Genetic analysis could not confirm a suspected ARVC although histopathological findings indicated clear fibro-fatty tissue infiltration with replacement of the myocardium in the right ventricle. Approximately $50 \%$ of ARVC cases remain negative after genetic testing. This might be explained by the complex genetic background of ARVC and low penetrance making it difficult to identify the true disease-causing variants accurately [43]. In summary, morphological abnormalities in the heart led to an ARVC suspicion during autopsy; however, genetic testing could not confirm this suspicion but indicated a possible disturbed ion channel function. In the end, both of them could have contributed to cardiac arrhythmias triggering her lethal cardiac infarction.

WES is one of the most commonly used new sequencing approaches and major advantages are a high throughput, well established sample preparation methods, and relatively low preparation and sequencing costs compared to traditional Sanger sequencing [44]. Additionally, sample preparation and sequencing can be performed from a small input amount of DNA, which can be crucial for post-mortem samples where the amount of available tissue and the DNA quality are limited. Library preparation and sequencing costs are comparable to a targeted gene approach, but the WES data analysis can be extended to the whole exome in order to identify new candidate genes in cases where the analysis remained negative within a predefined gene panel.

Significant challenges in WES represent the handling and processing steps of such huge amount of sequencing data, which requires a variety of computational and statistical approaches [45]. Consequently, sequencing data analysis and mainly interpretation are time-consuming and require expertise in the field to prevent false-positive or false-negative results. Although filtering strategies and protein prediction tools can help to interpret the pathogenicity of variants, pathological and functional consequences of variants often remain unclear and require further functional studies [46]. Negative genetic testing otherwise may be secondary to disease-causing sequence variants within genes not included in the cardiac gene panel, due to sequence alterations not detectable in WES approaches, such as deep intronic or splice mutations, or due to variants lost during bioinformatics steps rather than due to real absence of any sequence alterations. Accurate interpretation is even more difficult in complex multifactorial phenotypes such as in sudden unexplained death cases without any obvious morphological abnormalities, and lethality may be triggered by different events such as stress, drugs, or physical exertion [47].

The legal and ethical aspects of genetic analysis in forensic post-mortem investigations are complex, as the main forensic purpose is to determine the cause of death and not its implications for surviving relatives [48]. Therefore, genetic counselling of family members should be based on a multidisciplinary approach involving forensic pathologists, geneticists, and cardiologists to provide the family an opportunity to be informed in case of positive findings and offering them further clinical evaluation. Nowadays, minor preventive measures such as lifestyle modifications, prophylaxis, drug therapy, and implantable defibrillators are available in most cardiac diseases preventing another tragedy within the family [2]. In this study, genetic counselling of first-degree relatives was performed in two of the cases with potential diseasecausing variants (case III and case V), whereas the relatives of the other three cases were not available for counselling. Furthermore, functional studies to confirm the pathogenicity of found variants and family cosegregation analysis would have been crucial to verify the possible disease-causing impact of the observed variants, which are potential limitations of this study. DNA from family members of the five SUD cases was not available for co-segregation analysis, and hence, it is unknown if the observed variants were inherited from the parents or occurred de novo in the deceased.

In general, post-mortem genetic testing demonstrates an efficient strategy to investigate the cause of death in autopsy-negative sudden death cases. However, one of the major challenges remains the data management and variant interpretation of the huge amount of exome sequencing data and results should be regarded with caution.

Acknowledgments This project was supported by the Swiss National Science Foundation (SNF, project-Nr. 320030 149456). Special thanks to Luzy Bähr and Silke Feil for technical support, to Barbara Fliss for the 
colour images of the histological sections, and to Claudine Rieubland for additional information regarding the family history of case $\mathrm{V}$.

\section{Compliance with ethical standards}

Conflict of interest The authors declare that they have no competing interests.

Ethical approval All procedures performed in studies involving human participants were in accordance with the ethical standards of the institutional and/or national research committee and with the $1964 \mathrm{Hel}-$ sinki Declaration and its later amendments or comparable ethical standards.

\section{References}

1. Wren C, Sullivan JJ, Wright C (2000) Sudden death in children and adolescents. Heart 83:410-413

2. van der Werf C, van Langen IM, Wilde AA (2010) Sudden death in the young: what do we know about it and how to prevent? Circ Arrhythm Electrophysiol 3:96-104. doi:10.1161/CIRCEP.109. 877142

3. Ackerman MJ, Priori SG, Willems S, Berul C, Brugada R, Calkins H, Camm AJ, Ellinor PT, Gollob M, Hamilton R, Hershberger RE, Judge DP, Le Marec H, McKenna WJ, Schulze-Bahr E, Semsarian C, Towbin JA, Watkins H, Wilde A, Wolpert C, Zipes DP, Heart Rhythm S, European Heart Rhythm A (2011) HRS/EHRA expert consensus statement on the state of genetic testing for the channelopathies and cardiomyopathies: this document was developed as a partnership between the Heart Rhythm Society (HRS) and the European Heart Rhythm Association (EHRA). Heart Rhythm 8:1308-1339. doi:10.1093/europace/eur245

4. Tester DJ, Ackerman MJ (2012) The molecular autopsy: should the evaluation continue after the funeral? Pediatr Cardiol 33:461-470. doi:10.1007/s00246-012-0160-8

5. Virmani R, Burke AP, Farb A (2001) Sudden cardiac death. Cardiovasc Pathol 10:1039-1044

6. Hofer F, Fellmann F, Schläpfer J, Michaud K (2014) Sudden cardiac death in the young (5-39 years) in the canton of Vaud, Switzerland. Cardiovasc Disord 14:1471-2261

7. Cerrone M, Priori SG (2011) Genetics of sudden death: focus on inherited channelopathies. Eur Heart J 32:2109-2118. doi:10.1093/ eurheartj/ehr082

8. Narula N, Tester DJ, Paulmichl A, Maleszewski JJ, Ackerman MJ (2014) Post-mortem whole exome sequencing with gene-specific analysis for autopsy-negative sudden unexplained death in the young: a case series. Pediatr Cardiol. doi:10.1007/s00246-014$1082-4$

9. Abriel H, Zaklyazminskaya EV (2013) Cardiac channelopathies: genetic and molecular mechanisms. Gene 517:1-11. doi:10.1016/ j.gene.2012.12.061

10. Elliott P, Andersson B, Arbustini E, Bilinska Z, Cecchi F, Charron P, Dubourg O, Kühl U, Maisch B, McKenna WJ, Moserrat L, Pankuweit S, Rapezzi C, Seferovic P, Tavazzi L, Keren A (2008) Classification of the cardiomyopathies: a position statement from the European Society of Cardiology Working Group on Myocardial and Pericardial Diseases. Eur Heart J 29:270-276. doi:10.1093/ eurheartj/ehm342, 10.1093/eurheartj/ehm585

11. Campuzano O, Alcalde M, Berne P, Castro V, Guzzo G, Iglesias A, Alonso-Pulpon L, Garcia-Pavia P, Brugada J, Brugada R (2012) Genetic testing of candidate genes in arrhythmogenic right ventricular cardiomyopathy/dysplasia. Eur J Med Genet 55:225234. doi:10.1016/j.ejmg.2012.02.007

12. Loporcaro CG, Tester DJ, Maleszewski JJ, Kruisselbrink T, Ackerman MJ (2013) Confirmation of cause and manner of death via a comprehensive cardiac autopsy including whole exome nextgeneration sequencing. Arch Pathol Lab Med 138:1083-1089. doi: 10.5858/arpa.2013-0479-SA

13. Campuzano O, Sanchez-Molero O, Allegue C, Coll M, MademontSoler I, Selga E, Ferrer-Costa C, Mates J, Iglesias A, SarquellaBrugada G, Cesar S, Brugada J, Castella J, Medallo J, Brugada R (2014) Post-mortem genetic analysis in juvenile cases of sudden cardiac death. Forensic Sci Int 245C:30-37. doi:10.1016/j. forsciint.2014.10.004

14. Ng SB, Buckingham KJ, Lee C, Bigham AW, Tabor HK, Dent KM, Huff CD, Shannon PT, Jabs EW, Nickerson DA, Shendure J, Bamshad MJ (2010) Exome sequencing identifies the cause of a Mendelian disorder. Nat Genet 42:30-35. doi:10.1038/ng.499

15. Hertz CL, Christiansen SL, Ferrero-Miliani L, Fordyce SL, Dahl M, Holst AG, Ottesen GL, Frank-Hansen R, Bundgaard H, Morling N (2015) Next-generation sequencing of 34 genes in sudden unexplained death victims in forensics and in patients with channelopathic cardiac diseases. Int J Legal Med 129:793-800. doi:10.1007/s00414-014-1105-y

16. Behr E, Wood DA, Wright M, Syrris P, Sheppard MN, Casey A, Davies MJ, McKenna W (2003) Cardiological assessment of firstdegree relatives in sudden arrhythmic death syndrome. Lancet 362: 1457-1459. doi:10.1016/s0140-6736(03)14692-2

17. Stattin EL, Westin IM, Cederquist K, Jonasson J, Jonsson BA, Morner S, Norberg A, Krantz P, Wisten A (2015) Genetic screening in sudden cardiac death in the young can save future lives. Int $\mathrm{J}$ Legal Med. doi:10.1007/s00414-015-1237-8

18. Haghighi A, Tiwari A, Piri N, Nurnberg G, Saleh-Gohari N, Haghighi A, Neidhardt J, Nurnberg P, Berger W (2014) Homozygosity mapping and whole exome sequencing reveal a novel homozygous COL18A1 mutation causing Knobloch syndrome. PLoS One 9, e112747. doi:10.1371/journal.pone.0112747

19. Li H, Durbin R (2010) Fast and accurate long-read alignment with Burrows-Wheeler transform. Bioinformatics 26:589-595. doi:10. 1093/bioinformatics/btp698

20. Li H, Handsaker B, Wysoker A, Fennell T, Ruan J, Homer N, Marth G, Abecasis G, Durbin R, Genome Project Data Processing S (2009) The sequence alignment/Map format and SAMtools. Bioinformatics 25:2078-2079. doi:10.1093/bioinformatics/btp352

21. Wang K, Li M, Hakonarson H (2010) ANNOVAR: functional annotation of genetic variants from high-throughput sequencing data. Nucleic Acids Res 38, e164. doi:10.1093/nar/gkq603

22. Attanasio C, David A, Neerman-Arbez M (2003) Outcome of donor splice site mutations accounting for congenital afibrinogenemia reflects order of intron removal in the fibrinogen alpha gene (FGA). Blood 101:1851-1856. doi:10.1182/blood-2002-03-0853

23. Zeek PM (1942) Heart weight I. The weight of the normal human heart. Arch Pathol 34:820-832

24. Roden DM (2008) Long-QT syndrome. N Engl J Med 358:169176. doi:10.1056/NEJMcp0706513

25. Hayek E, Gring CN, Griffin BP (2005) Mitral valve prolapse. Lancet 365:507-518. doi:10.1016/s0140-6736(05)17869-6

26. Boileau C, Guo DC, Hanna N, Regalado ES, Detaint D, Gong L, Varret M, Prakash SK, Li AH, d'Indy H, Braverman AC, Grandchamp B, Kwartler CS, Gouya L, Santos-Cortez RL, Abifadel M, Leal SM, Muti C, Shendure J, Gross MS, Rieder MJ, Vahanian A, Nickerson DA, Michel JB, National Heart L, Blood Institute Go Exome Sequencing P, Jondeau G, Milewicz DM (2012) TGFB2 mutations cause familial thoracic aortic aneurysms and dissections associated with mild systemic features of Marfan syndrome. Nat Genet 44:916-921. doi:10.1038/ng.2348 
27. Van Norstrand DW, Asimaki A, Rubinos C, Dolmatova E, Srinivas M, Tester DJ, Saffitz JE, Duffy HS, Ackerman MJ (2012) Connexin 43 mutation causes heterogeneous gap junction loss and sudden infant death. Circulation 125:474-481. doi:10.1161/ CIRCULATIONAHA.111.057224

28. Gollob MH, Jones DL, Krahn AD, Danis L, Gong X-Q, Shao Q, Lui X, Veinot JP, Tang AS, Stewart AW, Tesson F, Klein GJ, Yee R, Skanes AC, Guiraudon GM, Ebihara L, Bai D (2006) Somatic mutations in the connexin 40 gene (GJA5) in atrial fibrillation. N Engl J Med 354:2677-2688

29. Takeshima H, Komazaki S, Nishi M, Iino M, Kangawa K (2000) Junctophilins: a novel family of junctional membrane complex proteins. Mol Cell 6:11-22

30. Beavers DL, Wang W, Ather S, Voigt N, Garbino A, Dixit SS, Landstrom AP, Li N, Wang Q, Olivotto I, Dobrev D, Ackerman MJ, Wehrens XH (2013) Mutation E169K in junctophilin-2 causes atrial fibrillation due to impaired RyR2 stabilization. J Am Coll Cardiol 62:2010-2019. doi:10.1016/j.jacc.2013.06.052

31. Olesen MS, Jensen NF, Holst AG, Nielsen JB, Tfelt-Hansen J, Jespersen T, Sajadieh A, Haunso S, Lund JT, Calloe K, Schmitt N, Svendsen JH (2011) A novel nonsense variant in Nav1.5 cofactor MOG1 eliminates its sodium current increasing effect and may increase the risk of arrhythmias. Can J Cardiol 27(523):e517-523. doi:10.1016/j.cjca.2011.01.003

32. Wu L, Yong SL, Fan C, Ni Y, Yoo S, Zhang T, Zhang X, ObejeroPaz CA, Rho HJ, Ke T, Szafranski P, Jones SW, Chen Q, Wang QK (2008) Identification of a new co-factor, MOG1, required for the full function of cardiac sodium channel Nav 1.5. J Biol Chem 283: 6968-6978. doi:10.1074/jbc.M709721200

33. Moss AJ (2002) Increased risk of arrhythmic events in long-QT syndrome with mutations in the pore region of the human ether-ago-go-related gene potassium channel. Circulation 105:794-799. doi:10.1161/hc0702.105124

34. Gong Q, Zhang L, Vincent GM, Horne BD, Zhou Z (2007) Nonsense mutations in hERG cause a decrease in mutant mRNA transcripts by nonsense-mediated mRNA decay in human long-QT syndrome. Circulation 116:17-24. doi:10.1161/ CIRCULATIONAHA.107.708818

35. Lin NC, Huang CL, Chen CY, Lin TY, Wang HY, Lu YH, Chen LM, Chen VC, Gossop M (2014) Effect of amphetamine on corrected-QT interval change during methadone maintenance treatment in Taiwan: a prospective cohort study. Drug Alcohol Rev 33: 194-201. doi:10.1111/dar.12099

36. van Noord C, Eijgelsheim M, Stricker BH (2010) Drug- and nondrug-associated QT interval prolongation. Br J Clin Pharmacol 70: 16-23. doi:10.1111/j.1365-2125.2010.03660.x

37. Crotti L, Hu D, Barajas-Martinez H, De Ferrari GM, Oliva A, Insolia R, Pollevick GD, Dagradi F, Guerchicoff A, Greco F, Schwartz PJ, Viskin S, Antzelevitch C (2012) Torsades de pointes following acute myocardial infarction: evidence for a deadly link with a common genetic variant. Heart Rhythm 9:1104-1112. doi: 10.1016/j.hrthm.2012.02.014

38. Crotti L, Lundquist AL, Insolia R, Pedrazzini M, Ferrandi C, De Ferrari GM, Vicentini A, Yang P, Roden DM, George AL Jr, Schwartz PJ (2005) KCNH2-K897T is a genetic modifier of latent congenital long-QT syndrome. Circulation 112:1251-1258. doi:10. 1161/CIRCULATIONAHA.105.549071

39. Kato K, Makiyama T, Wu J, Ding WG, Kimura H, Naiki N, Ohno S, Itoh H, Nakanishi T, Matsuura H, Horie M (2014) Cardiac channelopathies associated with infantile fatal ventricular arrhythmias: from the cradle to the bench. J Cardiovasc Electrophysiol 25:66-73. doi: $10.1111 /$ jce. 12270

40. Horigome H, Nagashima M, Sumitomo N, Yoshinaga M, Ushinohama H, Iwamoto M, Shiono J, Ichihashi K, Hasegawa S, Yoshikawa T, Matsunaga T, Goto H, Waki K, Arima M, Takasugi $\mathrm{H}$, Tanaka $\mathrm{Y}$, Tauchi N, Ikoma M, Inamura N, Takahashi $\mathrm{H}$, Shimizu W, Horie M (2010) Clinical characteristics and genetic background of congenital long-QT syndrome diagnosed in fetal, neonatal, and infantile life: a nationwide questionnaire survey in Japan. Circ Arrhythm Electrophysiol 3:10-17. doi:10.1161/ CIRCEP.109.882159

41. Gui J, Wang T, Trump D, Zimmer T, Lei M (2010) Mutationspecific effects of polymorphism H558R in SCN5A-related sick sinus syndrome. J Cardiovasc Electrophysiol 21:564-573. doi:10. 1111/j.1540-8167.2010.01762.x

42. Kauferstein S, Kiehne N, Peigneur S, Tytgat J, Bratzke H (2013) Cardiac channelopathy causing sudden death as revealed by molecular autopsy. Int J Legal Med 127:145-151. doi:10.1007/s00414012-0679-5

43. Iyer VR, Chin AJ (2013) Arrhythmogenic right ventricular cardiomyopathy/dysplasia (ARVC/D). Am J Med Genet C: Semin Med Genet. doi:10.1002/ajmg.c.31368

44. Morey M, Fernandez-Marmiesse A, Castineiras D, Fraga JM, Couce ML, Cocho JA (2013) A glimpse into past, present, and future DNA sequencing. Mol Genet Metab 110:3-24. doi:10. 1016/j.ymgme.2013.04.024

45. Stitziel NO, Kiezun A, Sunyaev S (2011) Computational and statistical approaches to analyzing variants identified by exome sequencing. Genome Biol 12. doi:10.1186/gb-2011-12-9-227

46. Koboldt DC, Steinberg KM, Larson DE, Wilson RK, Mardis ER (2013) The next-generation sequencing revolution and its impact on genomics. Cell 155:27-38. doi:10.1016/j.cell.2013.09.006

47. Killeen MJ (2009) Drug-induced arrhythmias and sudden cardiac death: implications for the pharmaceutical industry. Drug Discov Today 14:589-597. doi:10.1016/j.drudis.2009.03.004

48. Michaud K, Fellmann F, Abriel H, Beckmann JS, Mangin P, Elger BS (2009) Molecular autopsy in sudden cardiac death and its implication for families: discussion of the practical, legal and ethical aspects of the multidisciplinary collaboration. Swiss Med Wkly 139:712-718 